\title{
Receptive Fields of Motor Neurons Underlying Local Tactile Reflexes in the Locust
}

\author{
M. V. S. Siegler and M. Burrows \\ Department of Zoology, University of Cambridge, Cambridge CB2 3EJ, England
}

\begin{abstract}
The receptive fields of motor neurons to a hind leg were mapped by recording intracellularly from their cell bodies or from the muscle fibers they innervate while stimulating mechanoreceptors on the surface of that leg. Each motor neuron is affected by a specific array of receptors that make up its receptive field. Boundaries along the anteroposterior or dorsoventral axes of the leg divide the receptive fields into excitatory and inhibitory regions. Proximodistal boundaries may correspond to the articulations between parts of the leg. Motor neurons that innervate antagonistic muscles have complementary receptive fields, so that the region that is excitatory for one is inhibitory for the other. The receptive fields of the motor neurons overlap. Tactile stimulation therefore leads to a specific local reflex that involves the coordinated movement of the segments of a leg. Five local reflexes are described, each of which moves the leg away from the site of stimulation. Afferents from the external mechanoreceptors do not synapse directly on the motor neurons, but instead on spiking local interneurons, some of which then synapse directly on motor neurons. These local interneurons have smaller receptive fields delineated by the same boundaries, so that the receptive fields of the motor neurons can be constructed from appropriate combinations of them. It is suggested that receptive fields are organized as "functional maps" that are appropriate for particular behavioral responses rather than solely to preserve or refine spatial information.
\end{abstract}

Adjustments to the movements of a limb must be made during locomotion and posture to take account of changing loads of the body and changing aspects of the environment. Many of these changes will be experienced by an individual leg, which will therefore be called on to make its own adjustments. The implication is that the local neural circuitry of that leg must assess the sensory information and make the appropriate changes in its motor output to the muscles. Local interneurons must play a decisive role in these actions. Although these interneurons comprise the majority of neurons in most sophisticated nervous systems, it is in the insect that insights into their action are beginning to emerge.

In the locust, for example, two types of local interneuron, nonspiking and spiking, have been characterized within the segmental ganglion that controls the hind legs (Burrows and Siegler, 1984; Siegler and Burrows, 1979, 1984). The nonspiking local interneurons make direct inhibitory connections with some leg motor neurons, and excitatory connections with others (Burrows

Received May 20, 1985; revised July 22, 1985; accepted July 24, 1985.

This work was supported by an MRC. (IJK) project grant to M.V.S.S. and by NIH Grant NS16058 to M.B. We thank Peter Lawrence for helpful discussion during the course of the work, and Claire Hill-Venning, Michael Bate, Steve Buckingham, Joachim Pflüger, Peter Skorupski, and Alan Watson for many helpful comments on the manuscript.

Correspondence should be addressed to Dr. M. Burrows at the Department of Zoology, University of Cambridge, Downing Street, Cambridge CB2 3EJ, England.

Copyright $@ 1986$ Society for Neuroscience $0270-6474 / 86 / 020507-07 \$ 02.00 / 0$ and Siegler, 1976, 1978). They control the motor neurons in different combinations that correspond to their actions in a variety of motor patterns (Burrows, 1980). In addition, activation of the appropriate motor neurons is ensured by one-way inhibitory synapses between these interneurons (Burrows, 1979).

In contrast, spiking local interneurons play a major role in organizing sensory information from the legs. The different interneurons respond selectively to tactile and proprioceptive inputs. For example, some spike when particular regions of a leg are touched or when particular joints are moved (Burrows, 1985; Burrows and Siegler, 1982, 1984, 1985). Some of these interneurons make direct inhibitory synapses on motor neurons to mediate local compensatory reflexes (Burrows and Siegler, 1982; Siegler and Burrows, 1984).

The aim here is to account for the diversity within a population of spiking local interneurons and to suggest how the connections with sensory neurons may be viewed in a behavioral context. To do this, we have focused on the role of tactile inputs from the hind legs. Afferents from hairs make direct excitatory synapses on some spiking local interneurons (Siegler and Burrows, 1983). The combinations of hair inputs to the different spiking local interneurons are diverse but are organized in a topographical fashion (Burrows and Siegler, 1984, 1985). For example, one interneuron might be excited only by hairs on the antcrior surface of the femur and the tibia, whereas another might be excited by hairs on the ventral femur and inhibited by hairs on the dorsal tarsus (Burrows and Siegler, 1984, 1985). The picture is one of different but overlapping receptive fields for the individual spiking local interneurons within a population.

An expression of the importance of such a precise topographic representation of the hind leg in the population of spiking local interneurons might lie in the action of the leg motor neurons in movements elicited by stimulation of these external mechanoreceptors. In this paper we have mapped the receptive fields of many of the leg motor neurons and show that they differ in the extent and position of their excitatory and inhibitory regions, but that they may overlap. A touch to the leg results in the excitation of some motor neurons and the inhibition of others, in combinations that depend on the location of the receptors that are touched. By the preservation and appropriate redistribution of this topographical information, specific local reflex adjustments of a leg result from the stimulation of specific arrays of receptors.

\section{Materials and Methods}

Experiments were performed on adult male and female locusts Schistocerca gregaria (Forskal) from our crowded culture. Locusts were restrained ventral side uppermost, and, unless otherwise indicated, the legs were restrained at the coxal and femoral segments, but the tibiae and tarsi were free to move. The activity of motor neurons innervating muscles in a hind leg was recorded in three ways. First, selected muscles of one hind leg were implanted with pairs of $30 \mu \mathrm{m}$ stainless-steel wire electrodes, insulated except at the tip, to record extracellular activity. 


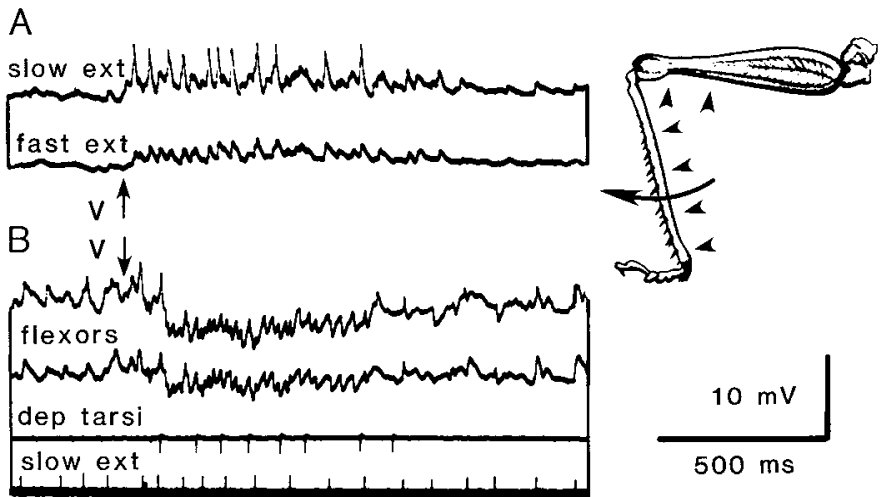

Figure 1. Extension of the tibia results from tactile stimulation of the ventral region of the femur and tibia. $A$, Intracellular recordings from the somata of the slow and fast extensor tibiae motor neurons (slow ext, fast ext). Touching the ventral ridges of the femur ( $v$, arrow) evokes a barrage of EPSPs in both extensor motor neurons and the slow extensor spikes several times. $B$, Intracellular recordings from the somata of two flexor tibiae motor neurons. Touching the ventral ridges of the femur $(v$, arrow) inhibits both. A slow depressor of the tarsus is recruited (dep tarsi, third trace), and the slow extensor tibiae motor neuron (fourth trace) increases its frequency of firing. The arrows indicate the onset of the tactile stimulus, which has approximately the same duration as the response of the motor neurons. The regions of the distal femur and the tibia where these effects may be evoked are indicated by the arrowheads in the drawing of a hind leg. The curved arrow indicates the direction of the evoked movement. Subsequent drawings follow this convention.

Second, recordings were made intracellularly from muscles that move the coxa, the trochantcr, or the tarsus. The selected muscles were exposed by removal of small regions of overlying cuticle. Third, intracellular recordings were made from the cell bodies of motor neurons in the metathoracic ganglion. Microelectrodes were filled with $2 \mathrm{M}$ potassium acetate and had DC resistances in saline of about $50 \mathrm{M} \Omega$. The meso- and metathoracic ganglia were exposed by a ventral dissection and were stabilized on a wax-covered platform. The body cavity was perfused continuously with saline (Usherwood and Grundfest, 1965). Just before intracellular recording, the thoracic ganglia were treated with a $0.2 \%$ solution of protease (Sigma Type VI) in saline for $4 \mathrm{~min}$.

Hairs (trichoid sensilla) and basiconic sensilla, which are the predominant mechanoreceptors on the surface of a leg, were stimulated in groups by touching a fine paint brush lightly to the surface of the cuticle or individually by deflecting them with a small insect pin. Campaniform sensilla, which are more sparsely distributed, rcspond to cuticular stress and are unlikely to have been activated by these light tactile stimuli. Moreover, the strength of a stimulus was such that it did not directly induce movements of the part of the leg being touched. Sensilla on the coxa, the proximal two-thirds of the femur, and the tarsus were not tested. It must be emphasized that we define the receptive field of a motor neuron only according to those receptors that were tested. Moreover, the reflex effects are elicited in the particular context of the experiments and might not find consistent expression in other contexts. For example, when the locust is standing with the ventral surface of the tarsus in contact with the ground, other reflex effects will be in competition.

Trichoid and basiconic sensilla are readily distinguished: The setae of basiconic sensilla are cone shaped and are only a small fraction of the length of those of the hairs; the basiconic scnsilla arc multiply innervated, whereas the hairs are singly innervated (Kendall, 1970; Siegler and Burrows, 1983). Hair afferents respond phasically to tactile stimuli (Pflüger, 1980; Runion and Usherwood, 1968; Siegler and Burrows, 1983). The response properties of a basiconic sensillum are not known, but at least one of its afferents is presumed to be mechanosensory, because touch can evoke avoidance movements of a leg.

Groups of sensilla that contribute to an excitatory or an inhibitory region of a receptive field were mapped according to their positions around the circumference of the leg and along its length. Receptive fields that are on either the dorsal or the ventral surface divide the circumference of the leg approximately in half along its anterior and posterior midline axes; those on either the anterior or posterior surface also divide

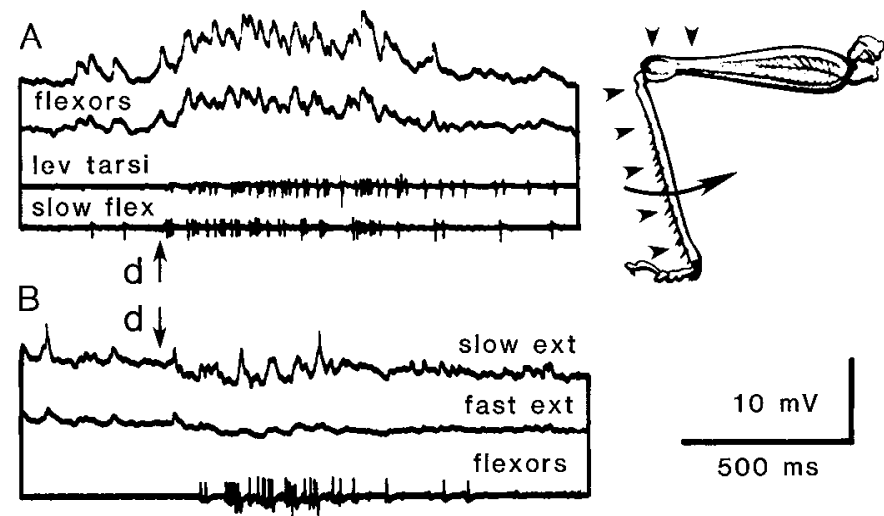

Figure 2. Flexion of the tibia results from tactile stimulation of the dorsal region of the femur and tibia. $A$, Intracellular recordings from the somata of two fast flexor tibiae motor neurons. Touching the dorsal surface of the distal femur ( $d$, arrow) evokes a barrage of EPSPs common to both neurons, increases the frequency of firing of slow flexor tibiae motor neurons (myogram fourth trace), and recruits the levator tarsi motor neuron (myogram third trace). $B$, Intracellular recordings from the somata of the slow and the fast extensor tibiae motor neurons. Touching the dorsal surface of the distal femur ( $d$, arrow) inhibits both motor neurons and excites flexor tibiae motor neurons (myogram third trace). The tibia therefore flexes, and peripheral feedback from this movement excites the slow extensor.

the circumference of the leg approximately in half, but along its dorsal and ventral midline axes. Along the length of the leg, boundaries of receptive fields occur at segment borders, or near other landmarks, such as the most proximal tibial spine. It was not feasible to map the receptive fields by specifying individually the sensilla that lie along the boundaries, for two reasons: First, in some regions a sensillum produces little or no effect on a motor neuron when it alone is activated; second, many sensilla may lie close to the boundaries, so that it is impossible to test them all.

The regions of the femur and tibia are as named by Albrecht (1953) and Bennet-Clark (1975). Muscle names and numbers are from Snodgrass (1929).

\section{Results}

Motor neurons that innervate muscles in a hind leg are excited or inhibited when hairs or basiconic sensilla on particular parts of that leg are touched. The effects on the motor neurons are reliable when measured by changes in their synaptic potentials but may be less reliably expressed as movements of the leg in contexts where they may conflict with other reflexes. All the effects we describe occur in the same behavioral context with the locust restrained and quiet. Motor neurons also respond to internal mechanoreceptors, but these effects are not considered here.

Each motor neuron can be considered to have a "receptive field," that is, an area of cuticle containing the sensilla to which it responds. Within a receptive field some regions are excitatory and some are inhibitory. The fields of the motor neurons overlap, so that tactile stimulation of a particular array of receptors evokes a movement of several joints of the leg. These movements are called tactile reflexes. They have a lower threshold for initiation and hence are distinct from resistance reflexes that mechanical stimulation can also evoke. The search for the receptive fields was limited to the femur and the tibia, but the motor neurons to be considered control movements about all joints of the leg. Depending on the region of cuticle that is touched, therefore, the tibia may be flexed or extended, the tarsus levated or depressed, the trochanter levated or depressed, and the coxa rotated anteriorly or posteriorly. These different reflex movements, and the receptive fields of the motor neurons, which underlie them, will be described in turn. 


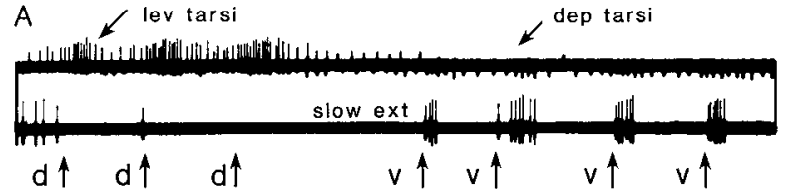

B

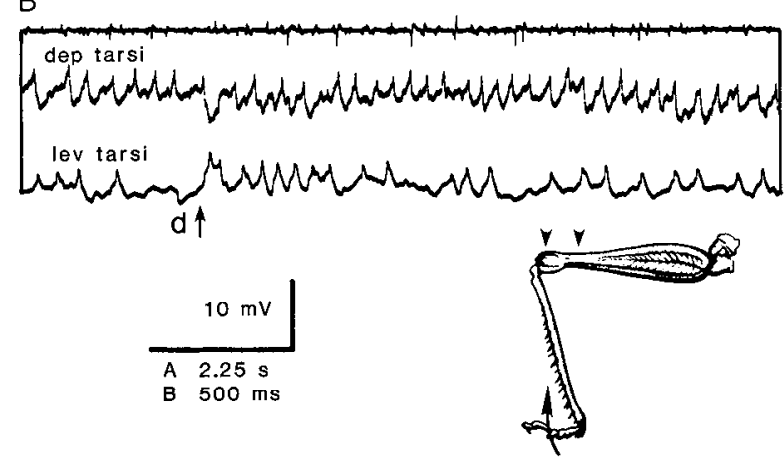

Figure 3. Levation of the tarsus results from tactile stimulation of the dorsal surface of the femur at its distal end. $A$, Extracellular myograms from the levator and depressor tarsi muscles (first trace) and the extensor tibiae muscle (second trace). Touching the dorsal surface of the distal femur ( $d$, arrow) excites the levator tarsi muscle (upward potentials). Touching the ventral surface of the tibia $(v$, arrow) excites slow motor neurons to the tarsal depressor muscle (downward potentials) and the slow extensor tibiae motor neuron. $B$, Intracellular recordings from a depressor tarsi motor neuron (second trace) and the levator tarsi (third trace) motor neuron. Touching the dorsal femur ( $d$, arrow) evokes a brief hyperpolarization of the depressor and a brief depolarization and increase in the frequency of spikes in the levator.

\section{Extension and flexion about the femorotibial joint}

When sensilla on the ventral femur or on the ventral tibia are touched, the tibia is extended about the femur (Fig. 1). The movement is caused by excitation of the extensor tibiae and inhibition of the flexor tibiae motor neurons. The slow and the fast extensor tibiae motor neurons are both depolarized by a barrage of common EPSPs that are sufficient to elicit a train of spikes from the slow motor neuron (Fig. 1A). The flexor motor neurons are hyperpolarized by a barrage of IPSPs (Fig. $1 B$ ) that are common to many of the nine excitatory motor neurons. These summed IPSPs suppress spikes in the tonically active slow flexor tibiae motor neurons and hyperpolarize the fast ones by some $4-6 \mathrm{mV}$ at their cell bodies. Recordings made simultaneously from pairs of extensor and flexor motor neurons do not reveal a correspondence of individual EPSPs in one with IPSPs in the other, indicating that the PSPs do not arise from the same presynaptic neurons.

This tactile reflex is of a lower threshold than the resistance reflex to imposed extension that operates at the femorotibial joint. If the touch applied to the ventral tibia is strong enough to impose an extension at the femorotibial joint, then the flexor tibiae motor neurons will be excited to resist the extension (Burrows and Horridge, 1974), instead of being inhibited.

When sensilla on the dorsal surface of the femur or tibia are touched, the tibia is flexed about the femur (Fig. 2). The movement is caused by excitation of the flexor tibiae and inhibition of the extensor tibiae motor neurons. Slow flexor motor neurons markedly increase their frequency of spikes and fast ones are depolarized by a barrage of common EPSPs (Fig. $2 A$ ). Both the slow and the fast extensor tibiae motor neurons are hyperpolarized, apparently as a result of the cessation of some excitatory inputs (Fig. $2 B$ ).

The receptive fields of the extensor tibiae motor neurons therefore consist of two regions: an excitatory region on the ventral surface of the femur at its distal end and the entire ventral
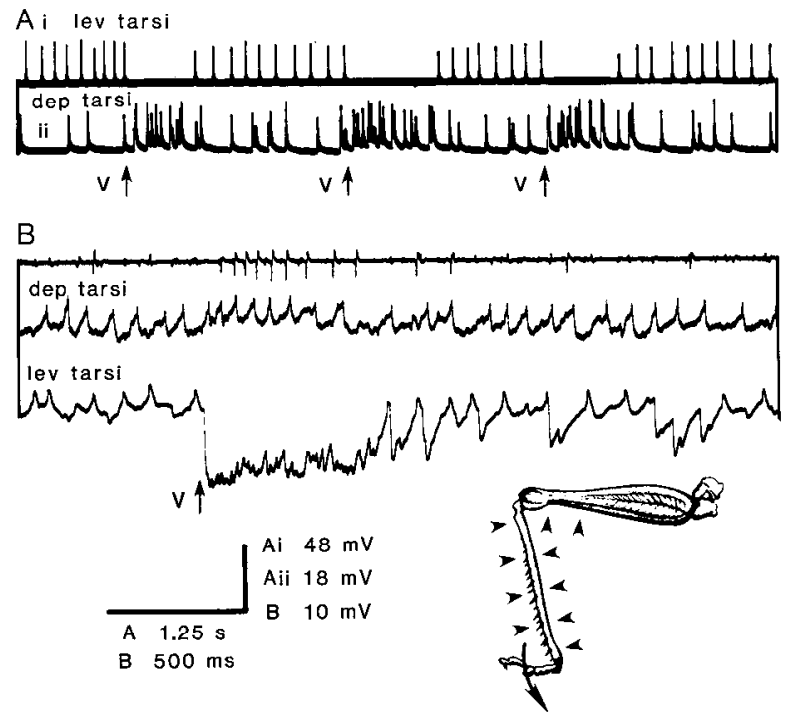

Figure 4. Depression of the tarsus results from tactile stimulation of the ventral surface of the femur at its distal end, or anywhere on the tibia. $A$, Intracellular recordings from fibers of the levator (first trace) and depressor tarsi muscles (second trace). Motor neurons to both muscles are spiking tonically at the beginning of the record. Touching the ventral surface of the femur ( $v$, arrow) inhibits EJPs in the levator and evokes a barrage of EJPs in the depressor. The femorotibial joint is fixed at an angle of $90^{\circ} . B$, Intracellular recordings from the somata of a depressor (second trace) and the levator tarsi (third trace) motor neurons. Touching the ventral femur ( $v$, arrow) increases the frequency of spikes in the impaled slow depressor and recruits another to spike (large downward potentials in the extracellular myogram, first trace). At the same time, the levator tarsi motor neuron is hyperpolarized by a barrage of large IPSPs, which continue at a lower frequency during the remainder of the record.

tibia, and an inhibitory region on the dorsal surface of the femur at its distal end and the entire dorsal tibia. The receptive fields of the flexor tibiae motor neurons also consist of two regions, which are opposite to those of the extensors. Thus, a touch to the ventral tibia excites extensors and inhibits flexors, whereas a touch to the dorsal tibia has the opposite effect.

\section{Levation and depression about the coxotrochanteral joint}

When sensilla on the dorsal femur are touched, the tarsus is levated about the tibia (Fig. 3). The movement is caused by a burst of spikes in the single levator tarsi motor neuron (Fig. $3 A$ ). Spikes in the depressor tarsi motor neurons are inhibited, as are those in the slow extensor tibiae. The synaptic events underlying these changes in the tarsal motor neurons are shown in Figure $3 B$. The levator is depolarized and the frequency of its spikes increases while a slow depressor is hyperpolarized by IPSPs that are no more than a few millivolts in amplitude.

When sensilla on the ventral surface of the femur or anywhere on the tibia are touched, the tarsus is depressed about the tibia (Fig. 4). The movement is caused by excitation of the depressor tarsi motor neurons and inhibition of the single levator tarsi (Fig. 4A). At the same time, the slow extensor tibiae motor neuron is excited (Fig. 3A). Deflecting individual hairs on the tibia or on the ventral margins of the femoral cover plates evokes a burst of excitatory junctional potentials (EJPs) from several motor neurons in the depressor muscle, while EJPs in the levator muscle are inhibited. On the ventral surface of the femur, however, it is usually necessary to touch a group of hairs to elicit a comparable response (Fig. $4 A$ ). The synaptic events underlying these changes in the motor neurons are shown in Figure $4 B$. A slow depressor tarsi motor neuron is depolarized and the frequency of its spikes increases, while the levator is hyperpolar- 

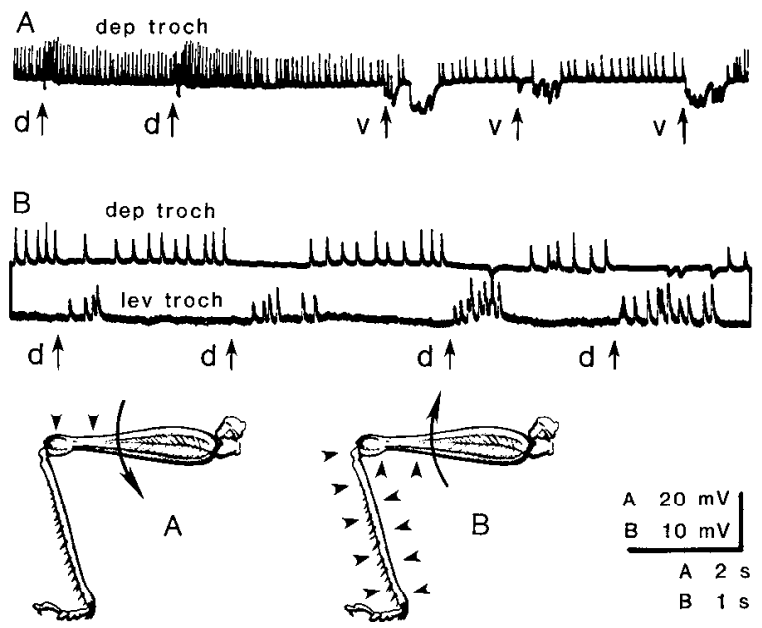

A $20 \mathrm{mV}$
B $10 \mathrm{mV}$

Figure 5. Local tactile reflexes for depression and levation of the trochanter. Recordings are made intracellularly from fibers of a trochanteral depressor (133a) and levator (131) muscle. $A$, Touching the dorsal surface of the distal femur ( $d$, arrow) excites excitatory motor neurons to the trochanteral depressor, whereas touching the ventral surface $(v$, arrow) inhibits them and recruits IJPs from a common inhibitory motor neuron, $\mathrm{CI}_{1}, B$, Touching the tibia dorsally (d, arrow) inhibits the depressor (first trace) and causes a train of EJPs in the levator. Touches in the series are successively longer-lasting, so that, with each, more EJPs occur in the levator and IJPs are recruited in the depressor.

ized. The IPSPs in the levator may continue at a low frequency for several seconds after the end of the stimulus.

The receptive field of the levator tarsi motor neuron therefore consists of two regions: an excitatory one on the dorsal surface of the femur at its distal end and an inhibitory one that includes the ventral surface of the femur at its distal end and the whole of the tibia. The receptive fields of the depressor tarsi motor neurons are the opposite of this.

The effects of touching sensilla are, however, complicated by the fact that active or passive movement of the femorotibial joint evokes strong compensatory reflex movements at the tibiotarsal joint (Burrows and Horridge, 1974). When the tibia is flexed, the tarsus is levated because the levator tarsi motor neuron is excited and the depressor tarsi motor neurons are inhibited. Conversely, when the tibia is extended, the tarsus is deprcssed because the depressor tarsi motor neurons are excited, and the levator tarsi is inhibited. In effect, the tarsus is kept at a constant angle with respect to the substrate during movements of the tibia.

The receptive fields of the tibial and the tarsal motor neurons overlap, and as a rcsult, a compcnsatory reflex as well as a tactile reflex movement of the tarsus can be evoked by the same stimulus. The compensatory reflex can either reinforce or override the tactile one, depending on which sensilla are touched. Touching the dorsal femur excites flexor tibiae motor neurons and the levator tarsi motor neuron. If the tibia flexes, the compensatory reflex of tarsal levation reinforces the tactile reflex. Touching the ventral femur excites the extensor tibiae and the depressor tarsi motor neurons. If the tibia extends, the compensatory reflex of tarsal depression again reinforces the tactile reflex. Such interactions could explain the prolonged excitation or inhibition of the levator tarsi motor neuron after touching the dorsal or the ventral surface of the femur, respectively. In contrast, touching the dorsal tibia may result in reflexes that would conflict. If the stimulus does not activate the flexor tibiae motor neurons, or if flexion is prevented, only the tactile reflex occurs: The levator is inhibited, and the depressors are excited. If, however, the tibia flexes, then the tactile reflex of tarsal depression is overridden by the stronger compensatory reflex in which the levator is excited and the depressors are inhibited.

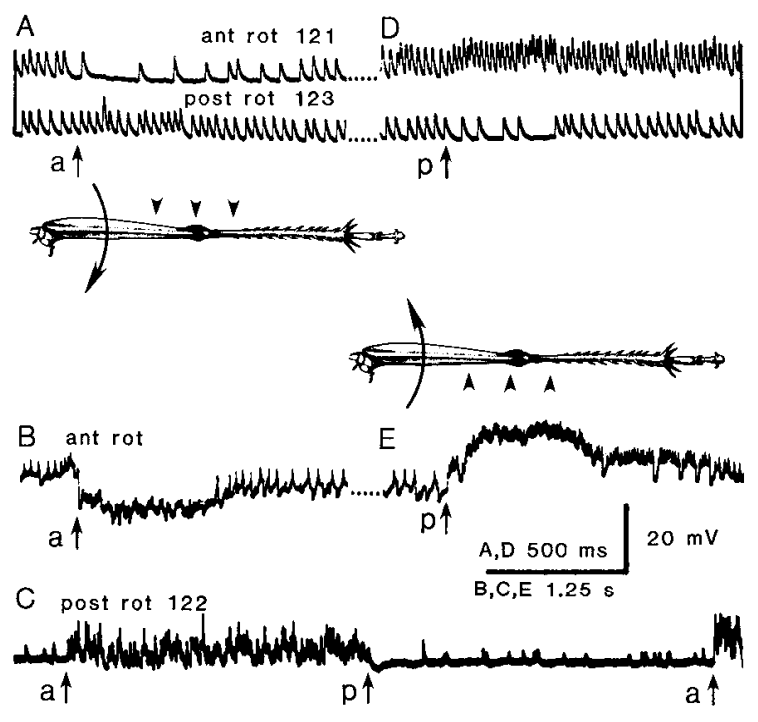

Figure 6. Local tactile reflexes for rotation of the coxa. $A$, Simultaneous intracellular recordings from fibers of the anterior (121) and a posterior rotator (123) of the coxa. Motor neurons to both muscles spike tonically. Touching the anterior surface of the femur ( $a$, arrow) reduces the frequency of EJPs in the anterior rotator but increases the frequency of EJPs in the posterior rotator. $B$, Intracellular recording from the soma of an anterior rotator motor neuron. Touching the anterior surface $(a$, arrow) of the distal femur evokes a barrage of IPSPs that hyperpolarize the motor neuron and inhibit the tonic spiking. $C$, Intracellular recordings from a fiber of another postcrior rotator muscle, 122. Touching the anterior surface of the femur ( $a$, arrow) evokes a barrage of EJPs, which continue until the posterior surface of the femur is touched $(p$, arrow). The motor neurons then spike only sporadically, until another touch to the anterior surface of the femur ( $a$, arrow). The effects on the motor neurons far outlast the duration of the stimulus. $D$, Touching the posterior surface of the femur ( $p$, arrow) increases the frequency of EJPs in the anterior rotator (121) but decreases their frequency in the posterior rotator (123). $E$, Touching the posterior surface of the distal femur evokes a large depolarization and increase in the frequency of spikes in an anterior rotator motor neuron. In the drawings, the hind leg is viewed dorsally, with its joints fully extended. The anterior surface of the leg is at the top.

\section{Levation and depression about the coxotrochanteral joint}

When sensilla on the dorsal surface of the femur are touched, the frequency of spikes increases in motor neurons that cause depression of the trochanter (Fig. 5A). Were the leg free to move, this response would result in depression of the femur about the coxa. The trochanter is fused to the femur, so that the trochanteral muscles move both segments.

When sensilla on the ventral surface of the femur, or anywhere on the tibia, are touched, motor neurons that cause levation of the trochanter are excited (Fig. $5 B$ ) and those causing depression are inhibited (Fig. 5). In addition, a common inhibitory motor neuron $\mathrm{CI}_{1}$ (Hale and Burrows, 1985) is excited, evoking a barrage of inhibitory junction potentials (IJPs) in the depressor muscle (Fig. 5A). Were the leg free to move, these responses would result in levation of the femur about the coxa.

The receptive ficlds of the trochanteral depressor motor neurons therefore consist of two regions: an excitatory region on the dorsal surface of the femur at its distal end and an inhibitory region that includes the ventral surface of the femur at its distal end and the whole of the tibia. The receptive fields of the levators are the opposite. These fields have the same boundaries as those of the tarsal motor neurons and also overlap with those of the tibial motor neurons. As a result, a touch to the dorsal surface of the femur near the femorotibial joint leads to depression of the femur, flexion of the tibia, and levation of the tarsus. 


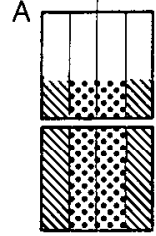

ext tibiae

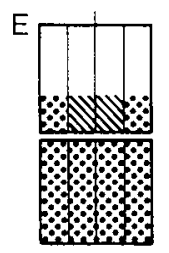

dep troch

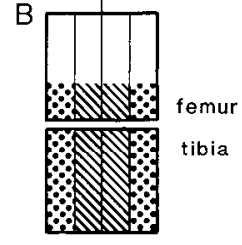

flex tibiae

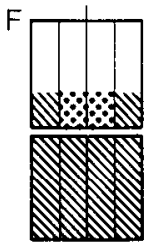

lev troch

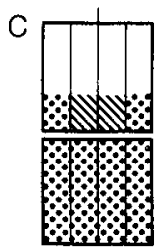

lev tarsi

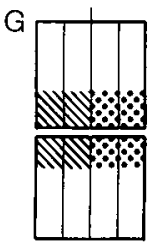

post rot coxa

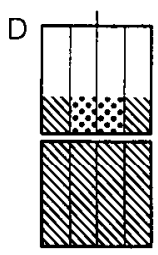

dep tarsi

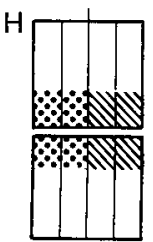

ant rot coxa
Figure 7. The receptive fields of some hind leg motor neurons. The diagrams represent the surface of the femur and the tibia of a hind leg opened by a longitudinal cut along the ventral midline and the femorotibial joint fully extended. The anterior half is on the left of the dorsal midline and the posterior half is on the right. On the femur, only sensilla on the distal part were tested; where responses are indicated, excitatory regions are cross-hatched, and inhibitory ones are stippled. Receptive fields: $A$, extensors tibiae; $B$, flexors tibiae; $C$, levator tarsi; $D$, depressors tarsi; $E$, depressors of the trochanter; $F$, levators of the trochanter; $G$, posterior rotators of the coxa; $H$, anterior rotators of the coxa. The receptive fields for the posterior and anterior rotators extend on the tibia only to the level of the first tibial spine.

\section{Anterior and posterior rotation of the coxa}

When sensilla are touched on the anterior surface of the femur at its distal end or on the anterior surface of the tibia proximal to the spines, there is an increase in the frequency of tonic EJPs in posterior rotator muscles of the coxa $(122,123,124)$ and a decrease in the frequency of, or cessation of, those in the anterior rotator muscle (121) (Fig. 6A). The motor neurons of muscle 123 show only a transient increase in frequency, whereas those of muscles 122 and 124 show a longer-lasting response. For example, touching the anterior surface of the femur initiates a barrage of EJPs in muscle 122 that continue until the posterior surface is touched (Fig. 6C). Intracellular recordings from an anterior rotator motor neuron to muscle 121 show that its tonic spikes are suppressed by a barrage of IPSPs (Fig. $6 B$ ). Were the leg free to move, these responses would result in the posterior rotation of the coxa, and thus the entire leg.

When sensilla are touched on the posterior surface of the equivalent regions of the femur and tibia, there is an increase in the frequency of tonic EJPs in the anterior rotator and a decrease in the frequency of EJPs in posterior rotators (Fig. 6D). The excitation of the anterior rotator motor neuron is caused by a barrage of summating EPSPs (Fig. $6 E$ ). Were the leg free to move, these responses would result in the anterior rotation of the coxa, and thus the entire leg.

The receptive fields of these coxal motor neurons are therefore organized according to an anteroposterior division of the leg, in contrast to those innervating muscles in the more distal segments, in which the receptive fields are organized according to a dorsoventral division. They again, however, consist of excitatory and inhibitory regions.

\section{Discussion}

\section{Organization of motor neuron receptive fields}

Several generalities emerge from a comparison of the receptive fields of hind leg motor neurons (Fig. 7). First, all the receptive fields contain both excitatory and inhibitory regions. This organization will accentuate differences in the effects from different
Table 1. Local tactile reflexes

\begin{tabular}{|c|c|c|c|}
\hline \multirow[b]{2}{*}{ Region of leg } & \multicolumn{3}{|l|}{ Movement } \\
\hline & $\begin{array}{l}\text { Trochanter/ } \\
\text { femur }\end{array}$ & Tibia & Tarsus \\
\hline Dorsal femur & Depression & Flexion & Levation \\
\hline Dorsal tibia & Levation & Flexion & Depression \\
\hline Ventral femur & Levation & Extension & Depression \\
\hline Ventral tibia & Levation & Extension & Depression \\
\hline Anterior femur, tibia & \multicolumn{3}{|c|}{ Posterior rotation of coxa } \\
\hline Posterior femur, tibia & \multicolumn{3}{|c|}{ Anterior rotation of coxa } \\
\hline
\end{tabular}

regions of a leg. For example, a flexor tibiae motor neuron is excited by touching the dorsal surface of the tibia and is inhibited by touching the ventral surface. The range over which the membrane potential of a motor neuron can be modulated during tactile stimulation is therefore greater than if its receptive field were solely excitatory or solely inhibitory. Second, motor neurons that innervate antagonist muscles have complementary receptive fields. For example, the dorsal tibia is excitatory for the flexor tibiae motor neurons but inhibitory for the extensors (Fig. 7, $A, B$ ). The tibia will flex when this region is stimulated, because the flexors are excited and because the extensors are inhibited. Third, the boundaries of the receptive fields are appropriate for the movements that are produced by particular motor neurons. For example, the coxal rotator motor neurons, which move the lcg anteriorly or postcriorly, have receptive fields that are divided into anterior and posterior regions (Fig. $7, G, H)$, whereas the extensor and flexor tibiae motor neurons, which move the tibia dorsally or ventrally, have receptive fields with dorsal and ventral regions (Fig. 7, $A, B$ ). Fourth, the receptive fields of the motor neurons that control different joints may be partially or wholly overlapping. As a result, motor neurons that control different segments of a hind leg are activated together in local reflexes that depend on the location of the surface sensilla that are stimulated (Table 1). Local tactile reflexes are, in effect, assembled from the overlap of the receptive fields of different motor neurons.

\section{Behavioral significance of the tactile reflexes}

The behavioral significance of the local reflexes can most readily be seen by depicting the movements that result from touching particular regions of a hind leg (Fig. 8, Table 1). By considering each in turn, it becomes clear that all are tactile avoidance reflexes.

1. When the ventral surface of the tibia or the femur is touched, the trochanter (and thus the femur) is levated, the tibia extended, and the tarsus depressed (Fig. 8A).

2. When the dorsal surface of the tibia is touched, the femur is again levated, but the tibia is now flexed (Fig. $8 B$ ). Flexion of the tibia will also elicit a compensatory levation of the tarsus and thus override the tactile response that would cause depression. A similar avoidance movement in response to touching hairs near the tibiotarsal joint occurs most readily in a freestanding locust if the tarsus is not touching the ground (Pflüger, 1980). If flexion is prevented, however, the same touch elicits depression of the tarsus and could stabilize the stance.

3. When the dorsal surface of the femur is touched, the femur is depressed, the tibia flexed, and the tarsus levated (Fig. 8C).

4. When the anterior surface of either the femur or the proximal tibia is touched, the coxal, and thus the entire leg will be rotated posteriorly (Fig. $8 D, \mathrm{i}$ ).

5. Conversely, when the posterior surface of either the femur or the proximal tibia is touched, the leg will be rotated anteriorly (Fig. $8 D$, ii).

These reflexes, as presented schematically in Figure 8, are 
A
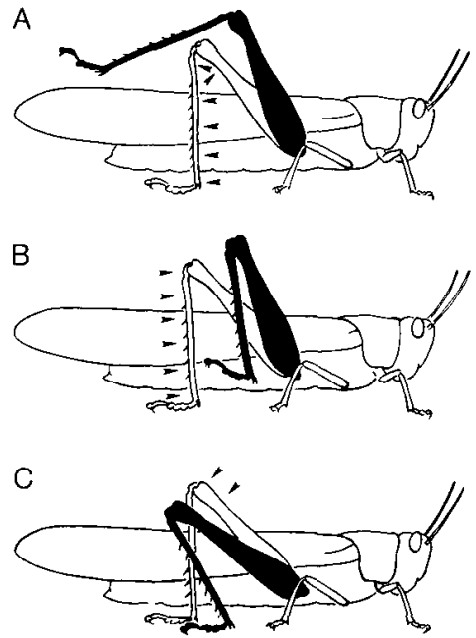

D

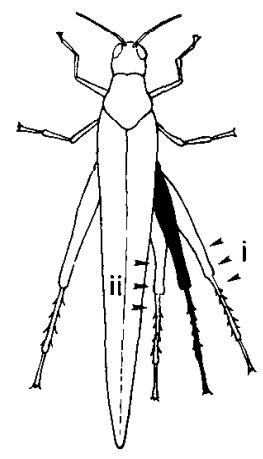

Figure 8. Local tactile avoidance reflexes of a hind leg in response to tactile stimuli. Each drawing of a locust shows the movements that would result from touching sensilla in the regions indicated by the arrows, as reconstructed from the receptive fields of Figure 7. The hind leg is initially in the position shown by the outline, and after the tactile stimulus, in the position shown by the silhouette. The reflexes depend on where the leg is touched, and move it away from the stimulus. The sensilla touched have the following locations: $A$, ventral surface of femur and tibia; $B$, dorsal surface of tibia; $C$, dorsal surface of femur; $D, i$, anterior surface of femur and proximal tibia; $D, i i$, posterior surface of femur and proximal tibia.

simplified compared with those that might normally occur. Consider, for example, the reflex response to touching hairs on the dorsal femur (Fig. 8C). The dorsal femur includes parts of the receptive fields of the anterior and posterior rotators of the coxa (Fig. 7, $G, H$ ). Thus, in addition to femoral depression, tibial flexion and tarsal levation, the leg will also be rotated anteriorly or posteriorly, depending on the position of the stimulated hairs.

The importance of the position of the mechanoreceptors in evoking particular local reflex movements of a leg is a new observation. It does, however, accord with previous descriptions of specific movements elicited by stimulation of external mechanoreceptors. More complex behavior such as stereotyped grooming movements by a leg are elicited by touching hairs or bristles on the compound eyes of a cricket (Honegger et al., 1979), on the prothoracic spine of a locust (Rowell, 1969) and on the notum and tegula of Drosophila (Vandervorst and Ghysen, 1980). In pupal moths, hairs within the gin trap and others at the same level along the anterior-posterior axis of the body elicit closure of the gin trap (Bate, 1973). Avoidance movements of a leg occur when hairs or bristles on that leg are touched in cockroaches (Pringle, 1940), locusts (Pflüger, 1980, Runion and Usherwood, 1968), and Drosophila (Vandervorst and Ghysen, 1980). In Drosophila, the behavioral responses differ according to the location of the sensilla that are touched. Bristles at some positions on the notum and tegula elicit grooming by the prothoracic leg, others grooming by the metathoracic leg (Vandervorst and Ghysen, 1980). Similarly, bristles on the anterior femur of the prothoracic leg elicit withdrawal of that leg, whereas those on the posterior femur elicit grooming by the mesothoracic leg. The present results show an even greater specificity in the behavioral responses of the locust. Specific movements of a hind leg are elicited according to whether the sensilla are on the tibia or the femur and whether they are dorsal or ventral, anterior or posterior, and proximal or distal.

\section{Neural circuitry of the tactile reflexes}

The afferents from the hairs on the hind leg do not connect directly with motor neurons, but instead first make excitatory connections with spiking local interneurons (Siegler and Bur-

rows, 1983). The afferents from several hairs on a particular region of cuticle converge on one interneuron, forming the excitatory region of its receptive field. Conversely, an afferent may make divergent connections with a group of interneurons and thus will contribute to the receptive field of each. Some interneurons have receptive fields with excitatory regions that overlap each other completely, whereas others have fields with little or no overlap (Burrows and Siegler, 1985).

Spiking local interneurons may connect directly with motor neurons. For example, two identified spiking local interneurons are responsible for the large IPSPs that occur in the levator tarsi motor neuron, in response to tactile stimuli to the tibia (Fig. 4), or when the tibia is extended about the femur (Burrows and Siegler, 1982). These are, however, but a few of the many spiking local interneurons within the metathoracic ganglion whose morphology and sensory inputs have been described (Burrows, 1985; Burrows and Siegler, 1984, 1985; Siegler and Burrows, 1984). Those described so far have their cell bodies in one of two clusters, which together contain some 140 cell bodies for each half of the ganglion. The problem of tracing connectivity is thus largely one of numbers: It is necessary to match these interneurons with the 50 or so motor neurons that innervate the muscles of a hind leg. Further indirect though persuasive evidence for the involvement of the spiking local interneurons in these reflexes is that their receptive fields are delineated by the same anatomical boundaries as are those of the motor neurons (Burrows and Siegler, 1985). The receptive fields of the interneurons are, in general, smaller than those of the motor neurons, but given the appropriate conncctions between interncurons and motor neurons, the fields of the motor neurons can be "assembled" from those of the interneurons.

Some aspects of the connectivity of the interneurons presynaptic to the motor neurons can be inferred from the present results. First, the interneurons that mediate the excitatory part of a reflex (for example, excitation of extensor tibiae motor neurons) are not the same ones that mediate the inhibitory part (that is, the concurrent inhibition of the flexors). During tactile stimulation, the EPSPs in an extensor do not correspond onefor-one with the IPSPs in a flexor motor neuron. This accords with previous findings, where IPSPs in the levator tarsi motor neuron evoked by two spiking local interneurons were not correlated with EPSPs in antagonist depressor tarsi motor neurons or with IPSPs in other motor neurons that are usually coactivated (Burrows and Siegler, 1982). Second, the motor neurons that innervate the same muscle receive synaptic inputs in common during tactile stimulation. The spiking local interneurons would not, therefore, necessarily have restricted outputs, as might be inferred from the finding that some connected only with a single motor neuron (Burrows and Siegler, 1982). Instead, the rule may be that the interneurons connect with the pool of motor neurons of a particular muscle, which may be as many as nine or as few as one.

\section{Origin and importance of receptive field houndaries}

The sensory afferents must encode spatial information if the central nervous system is to assemble the receptive fields of motor neurons and interneurons (Burrows and Siegler, 1985). On the distal femur, for example, spatial information from afferents must be sufficient to distinguish four sectors. Afferents must be specified as either dorsal or ventral, to generate the receptive fields of some motor neurons (Fig. $7, A-F$ ), and as either anterior or posterior, to generate the receptive fields of others (Fig. 7, $G, H$ ). On the tibia the afferents must be specified even more precisely. They must be specified as proximal or distal to the first tibial spines to generate the receptive fields of the coxal rotator motor neurons (Fig. 7, $G, H$ ) and some spiking local interneurons (e.g., Figs. 6-1 to 6-6 of Burrows and Siegler, 1985 ), and proximal or distal to the last tibial spines to generate the receptive fields of some other spiking local interneurons (e.g., 
Figs. 6-12 to 6-14 of Burrows and Siegler, 1985). Within each of these three proximodistal sectors are the four circumferential ones found also on the femur. Thus, a total of 12 sectors must be specified on the tibia.

How is this spatial information derived? Vandervorst and Ghysen (1980) suggest that the position of bristles on the legs of Drosophila is specified according to the anteroposterior compartment boundaries that operate during epidermal development. The divisions between the regions of bristles that evoke different behavioral responses appear to coincide with those of the compartment boundaries. Compartmental lineage is thus proposed to be the determinant of sensory connections and, ultimately, of behavioral responses. To account for the majority of receptive fields of motor neurons and spiking local interncurons in locusts, such a model would, however, require additional boundaries along the leg and a division into dorsal and ventral compartments. In Drosophila, the wings are divided initially during development into anterior and posterior compartments, and later into dorsal and ventral ones (Morata and Lawrence, 1977). Evidence for dorsal and ventral compartments of the legs is, however, equivocal (Lawrence et al., 1979; Steiner, 1976). A compartmental basis for the behavioral responses in Drosophila is further open to question because the compartmental identities of bristles were not determined in the same animals as those in which the behavioral responses were tested. This is a problem because bristles in the rows near a boundary can vary in their compartmental identity (Lawrence et al., 1979).

The results of grafting and regeneration experiments on cockroach legs indicate that positions of sensilla on the cuticle are encoded with considerably more specificity than that derived from their compartmental lineages. French cites his own results $(1978,1980)$ and those of Bohn $(1967,1970)$ and Bullière $(1970)$ to show that position is mapped as a continuous sequence of values, both around the circumference and along the length of a leg. Some further evidence indicates a division of the leg into anterior and posterior compartments as in Drosophila (French, 1980 ). While a continuum of values can provide precise information about the position of sensilla on a leg, it does not account for the origin of boundaries within and between receptive fields. Boundaries imply discontinuities, rather than a continuum. These could arise in the labeling of sensory afferents or in the reading of a continuum of labels by postsynaptic neurons during the development of the central nervous system.

\section{Receptive fields provide functional maps}

Whatever the mechanism, it is clear that spatial information is encoded in the receptive fields of motor neurons and spiking local interneurons. The role of a receptive field is not simply to "tell" the locust where its leg has been touched. More importantly, a receptive field delineates regions of sensilla that evoke particular behavioral responses. The hair afferents are sorted onto interneurons to construct functional maps, not solely to preserve spatial information. It is from combinations of these maps that the receptive fields of motor neurons are constructed. The spatial information is therefore assembled according to functional constraints with specific regard to the behavior of the animal.

\section{References}

Albrecht, F. O. (1953) The Anatomy of the Migratory Locust, Athlone Press, London.

Bate, C. M. (1973) The mechanism of the pupal gin trap. I. Segmental gradients and the connexions of the triggering sensilla. J. Exp. Biol. 59: 95-107.

Bennet-Clark, H. C. (1975) The energetics of the jump of the locust, Schistocerca gregaria. J. Exp. Biol. 63: 53-83.

Bohn, H. (1967) Transplantationsexperimente mit interkalarer Regeneration zum Nachweis eines sich segmental wiederholenden Gradienten im Bein von Leucophaea (Blattaria). Verh. Dtsch. Zool. Ges. Gottingen Suppl. 30: 499-508.
Bohn, H. (1970) Interkalare Regeneration und segmentale Gradienten bei den Extremitäten von Leucophaea-Larven (Blattaria). I. Femur und Tibia. Wilhelm Roux Arch. 165: 303-341.

Bullière, D. (1970) Sur la déterminisme de la qualité régionale des régénérates d'appendices chez la Blatte, Blabera craniifer. J. Embryol. Exp. Morphol. 23: 323-335.

Burrows, M. (1979) Graded synaptic transmission between local premotor interneurons of the locust. J. Neurophysiol. 42: 1108-1123.

Burrows, M. (1980) The control of sets of motoneurones by local interneurones in the locust. J. Physiol. (Lond.) 298: 213-233.

Burrows, M. (1985) The processing of mechanosensory information by spiking local interneurons in the locust. J. Neurophysiol. 54: 463478.

Burrows, M. and G. A. Horridge (1974) The organization of inputs to motoneurones of the locust metathoracic leg. Phil. Trans. R. Soc. Lond. [Biol.] 269: 49-94.

Burrows, M., and M. V. S. Siegler (1976) Transmission without spikes between locust interneurones and motoneurones. Nature 262: 222224.

Burrows, M., and M. V.S. Siegler (1978) Graded synaptic transmission between local interneurones and motor neurones in the metathoracic ganglion of the locust. J. Physiol. (Lond.) 285: 231-255.

Burrows, M., and M. V. S. Siegler (1982) Spiking local interneurons mediate local reflexes. Science 217: 650-652.

Burrows, M., and M. V. S. Siegler (1984) The morphological diversity and receptive fields of spiking local interneurones in the locust metathoracic ganglion. J. Comp. Neurol. 224: 483-508.

Burrows, M., and M. V.S. Siegler (1985) The organization of receptive fields of spiking local interneurons in the locust with inputs from hair afferents. J. Neurophysiol. 53: 1147-1157.

French, V. (1978) Intercalary regeneration around the circumference of the cockroach leg. J. Embryol. Exp. Morphol. 47: 53-84.

French, V. (1980) Positional information around the segments of the cockroach leg. J. Embryol. Exp. Morphol. 59: 218-313.

Hale, J. P., and M. Burrows (1985) Innervation patterns of inhibitory motor neurones in the thorax of the locust. J. Exp. Biol. 117: 401413.

Honegger, W. H., H. Reif, and W. Muller (1979) Sensory mechanism of eye cleaning behavior in the cricket Gryllus campestris. J. Comp. Physiol. 129: 247-256.

Kendall, M. D. (1970) The anatomy of the tarsi of Schistocerca gregaria Forskal. Z. Zellforsch. 109: 112-137.

Lawrence, P. A., G. Struhl, and G. Morata (1979) Bristle patterns and compartment boundaries in the tarsi of Drosophila. J. Embryol. Exp. Morphol. 51: 195-208.

Morata, G., and P. A. Lawrence (1977) Homeotic genes, compartments and cell determination in Drosophila. Nature 265: 211-216.

Pflüger, H-J. (1980) The function of hair sensilla on the locust's leg: the role of tibial hairs. J. Exp. Biol. 87: 163-175.

Pringle, J. W. S. (1940) The reflex mechanism of the insect leg. J. Exp. Biol. 17: 8-17.

Rowell, C. H. F. (1969) The control of reflex responsiveness and the integration of behaviour. In The Physiology of the Insect Central Nervous System, J. E. Treherne and J. W. L. Beament, eds., pp. 157164, Academic, London.

Runion, H. I., and P. N. R. Usherwood (1968) Tarsal receptors and leg reflexes in the locust and grasshopper. J. Exp. Biol. 49: 421-436.

Siegler, M. V. S., and M. Burrows (1979) The morphology of local non-spiking interneurones in the metathoracic ganglion of the locust. J. Comp. Neurol. 183: 121-148.

Siegler, M. V. S., and M. Burrows (1983) Spiking local interneurons as primary integrators of mechanosensory information in the locust. J. Neurophysiol. 50: 1281-1295.

Siegler, M. V. S., and M. Burrows (1984) The morphology of two groups of spiking local interneurones in the metathoracic ganglion of the locust. J. Comp. Neurol. 224: 463-482.

Snodgrass, R. E. (1929) The thoracic mechanism of a grasshopper, and its antecedents. Smithson. Misc. Collect. 82: 1-111.

Steiner, E. (1976) Establishment of compartments in the developing leg imaginal discs of Drosophila melanogaster. Wilhelm Roux Archiv. 180: 9-30.

Usherwood, P. N. R., and H. Grundfest (1965) Peripheral inhibition in skeletal muscle of insects. J. Neurophysiol. 28: 497-518.

Vandervorst, P., and A. Ghysen (1980) Genetic control of sensory connections in Drosophila. Nature 286: 65-67. 